\title{
RELAÇÃO ENTRE QUALIDADE DE VIDA, ESTRESSE E TRABALHO ENTRE ESTUDANTES DE FISIOTERAPIA
}

\author{
Christiane Athayde SANTOS ${ }^{1}$ \\ Núbia Ceci Pereira GOMES ${ }^{2}$ \\ Ana Mônica Macedo de OLIVEIRA ${ }^{3}$ \\ Danilo Lima CARREIRO ${ }^{4}$ \\ Laura Tatiany Mineiro COUTINHO 5 \\ Wagner Luiz Mineiro COUTINHO
}

1. Pós-graduada em Fisioterapia aplicada a Traumatologia e Ortopedia pelo Centro Universitário do Triângulo (UNITRI). Professora da Associação Educativa do Brasil (SOEBRAS), chrisataydesantos@yahoo.com.br

2. Fisioterapeuta graduada pela SOEBRAS,

nubiaceci@hotmail.com

3. Fisioterapeuta graduada pela SOEBRAS,

anamonica1969@yahoo.com.br

4. Mestre em Ciências da Saúde pela Universidade Estadual de Montes Claros (Unimontes). Professor das Faculdades

Santo Agostinho (FASA) e da SOEBRAS, danilolimacarreiro@gmail.com

5. Mestre em Ciências da Saúde pela Unimontes. Professora da SOEBRAS, mineiro.laura@ gmail.com

6.Mestre em Ciências da Saúde pela Unimontes. Professor da SOEBRAS, coutinhowlm@gmail.com

\section{Recebido em: 30/05/2014 - Aprovado em: 30/06/2014 - Disponibilizado em: 30/07/2014}

RESUMO: este estudo teve por objetivo determinar a relação entre percepção do nível de qualidade de vida (QV) com estresse e trabalho entre estudantes de fisioterapia. Trata-se de um estudo transversal analítico no qual se utilizaram para coleta de dados: WHOQOL-Bref, Inventário de Sintomas de Stress de Lipp, protocolo de avaliação de atividade física preconizada pela Organização Mundial de Saúde e pelo Ministério da Saúde do Brasil, Critério de Classificação Econômica Brasil e Questionário demográfico-socioeconômico, condições laborais e discentes. Registrou-se menor chance de baixa percepção no nível de QV geral entre estudantes do turno diurno e maior chance entre os estudantes com sinais de estresse na fase de resistência. Maior chance de baixa percepção no nível de QV no domínio físico entre os estudantes com sinais de estresse nas fases de resistência e exaustão. Menor chance de baixa percepção no nível de QV no domínio psicológico entre estudantes que relataram não ter filho e maior chance entre estudantes com sinais de estresse na fase de resistência. Menor chance de baixa percepção no nível de QV no domínio ambiental entre estudantes que não trabalham e maior chance entre estudantes com sinais de estresse nas fases de alerta e resistência.

Palavras-chave: Qualidade de vida. Estresse ocupacional. Trabalho. Centros médicos acadêmicos. Fisioterapia.

\section{RELATIONSHIP BETWEEN QUALITY OF LIFE, STRESS AND WORK AMONG STUDENTS OF PHYSIOTHERAPY}

\begin{abstract}
This study aimed to determine the relationship between perceived level of quality of life (QL) with stress and work among students of physiotherapy. This is an analytical cross-sectional study in which it was used for data collection: WHOQOL-Bref, Inventory of Stress Symptoms Lipp, evaluation protocol of physical activity recommended by the World Health Organization and the Ministry of Health of Brazil, Critério de Classificação Econômica Brasil and socioeconomic - demographic questionnaire, students and labor conditions. It was registered less chance of perception in the low level of overall QL between students in the day shift and greater chance among students with signs of stress in the resistance stage. Greater chance of low level perception of QL in the physical domain among students with signs of stress in the stages of resistance and exhaustion. Less chance of low level perception level of QL in the psychological domain among students who reported not having a child and greater chance among students with signs of stress in the resistance stage. Less chance of low perception level of QL in the environmental field between students who don't work and greater chance among students with signs of stress in the stages of alertness and resistance.
\end{abstract}

Keywords: Quality of life. Occupational stress. Work. Academic medical centers. Physiotherapy.

\section{INTRODUÇÃO}

Qualidade de vida (QV) é um termo sem definição consensual que pode associar- se a aspectos relacionados ao ser humano e à sua perspectiva subjetiva de satisfação quanto à vida familiar, amorosa, social, ambiental e 
existencial. No campo da saúde, a percepção do nível de QV tem conotação de estilo de vida saudável ou de superação dos estados e condições de morbidade, podendo esta ser impactada por fatores psicológicos e físicos. (FERNANDES, 1996; MINAYO, HARTZ e BUSS, 2000; MACIEL, 2006).

Dentre os fatores psicológicos, por causar um estado de tensão com consequências na homeostase orgânica, o estresse é compreendido como reações do organismo frente uma nova situação que exige da pessoa esforços de adaptação. (CALAIS et al., 2007; BUBLITZ et al., 2012). Ao se considerar o ambiente acadêmico se destacam como fatores estressores: espaço competitivo e demarcado por ansiedade, responsabilidades, grande número de atividades acadêmicas, dificuldades financeiras e tensão em relação à escolha profissional. (BOTTI, LIMA e SIMÕES, 2010).

Neste contexto acadêmico, o estudante trabalhador pode mostrar-se ainda mais susceptível aos agentes estressores e aos possíveis impactos negativos na percepção da QV, uma vez que além da exposição aos agentes estressores mencionados soma-se ainda, o fato do estudante trabalhador parecer ter no estudo uma extensão da sua jornada de trabalho. (TOMBOLATO, 2005).

Desta forma este estudo objetivou determinar a relação entre percepção do nível de QV, estresse e trabalho entre estudantes de fisioterapia.

\section{METODOLOGIA}

Trata-se de um estudo transversal analítico realizado em uma instituição de ensino superior em Montes Claros, Minas Gerais. A população constituiu-se por estudantes com idade acima de 18 anos, matriculados no curso de graduação em fisioterapia, no primeiro semestre letivo de 2013. De acordo com dados da instituição envolvida na pesquisa, o número de estudantes correspondia a 427 sujeitos. No cálculo amostral considerou-se a estimativa para proporções da ocorrência dos eventos em $50 \%$ da população, nível de confiança de $95 \%$, erro de $4 \%$ e taxa de não resposta de $20 \%$ identificando-se a partir de tais critérios a necessidade da participação de 299 sujeitos. Durante a coleta de dados, considerou-se a presença do estudante em sala de aula e desta forma a a/mostra final foi composta por 310 sujeitos.

Os dados foram coletados por pesquisadores previamente treinados, sob a supervisão de psicóloga envolvida no estudo, que também se responsabilizou pela avaliação dos questionários. Não foram permitidas conversas durante a aplicação dos questionários e, também foi estabelecida uma distância mínima de $0,50 \mathrm{~cm}$ entre os sujeitos a fim de resguardar a privacidade ao responder o protocolo de pesquisa. 
Considerou-se como variável et al., 2000). As variáveis independentes dependente, a percepção do nível de QV, foram classificadas em individuais avaliada pelo WHOQOL-bref. Trata-se de um (demográficas e socioeconômicas); condições questionário validado para uso no Brasil, de saúde, discentes e de trabalho.

$\mathrm{Na}$ Tabela 1 são apresentadas as constituído por 26 itens que envolvem os domínios físico, psicológico, social e categorizações das variáveis em estudo. ambiental, no qual maior escore corresponde à melhor percepção do nível de QV. (FLECK

Tabela 1 - Categorizações das variáveis, relação entre qualidade de vida, estresse e trabalho entre estudantes de fisioterapia, Montes Claros - MG, 2013 (n=300)

\begin{tabular}{|c|c|c|}
\hline Variáveis & Categorização & $\begin{array}{c}\text { Referência } \\
\text { categorização }\end{array}$ \\
\hline \multicolumn{3}{|l|}{ Dependentes } \\
\hline Percepção nível QV geral $^{\mathrm{a}}$ & $0=$ alta $1=$ baixa & $(\text { menor quartil })^{\mathrm{b}}$ \\
\hline Percepção nível QV domínio físico ${ }^{\mathrm{a}}$ & $0=$ alta $1=$ baixa & $(\text { menor quartil })^{\mathrm{b}}$ \\
\hline Percepção nível QV domínio psicológico ${ }^{\mathrm{a}}$ & $0=$ alta $1=$ baixa & $(\text { menor quartil })^{\mathrm{b}}$ \\
\hline Percepção nível QV domínio social ${ }^{\mathrm{a}}$ & $0=$ alta $1=$ baixa & (menor quartil) $)^{\mathrm{b}}$ \\
\hline Percepção nível QV domínio ambiental $^{\mathrm{a}}$ & $0=$ alta $1=$ baixa & $\left(\right.$ menor quartil) ${ }^{\mathrm{b}}$ \\
\hline \multicolumn{3}{|l|}{ Independentes } \\
\hline \multicolumn{3}{|l|}{ Individuais } \\
\hline \multicolumn{3}{|l|}{ Demográficas } \\
\hline Sexo & $0=$ masculino $1=$ feminino & $\mathrm{c}, \mathrm{d}$ \\
\hline Idade & $0=\leq 22$ anos $1=>22$ anos & média $^{c}$ \\
\hline Raça ou cor da pele $e^{e}$ & $0=$ branca $/$ amarela $1=$ preta $/$ parda & - \\
\hline \multicolumn{3}{|l|}{ Socioeconômicas } \\
\hline Estado civil $^{\mathrm{e}}$ & $0=$ casado $1=$ solteiro/divorciado/desquitado ${ }^{\mathrm{f}}$ & $\mathrm{c}$ \\
\hline Filhos & $0=\operatorname{sim} 1=$ não & - \\
\hline Trabalha & $0=\operatorname{sim} 1=$ não & $\mathrm{d}$ \\
\hline Segmentação econômica ${ }^{\mathrm{g}}$ & $0=\operatorname{alta}(\mathrm{A} 1 / \mathrm{B} 2) 1=$ baixa $(\mathrm{C} 1 / \mathrm{E})$ & $\mathrm{h}$ \\
\hline \multicolumn{3}{|l|}{ Condições de saúde } \\
\hline Sinais de estresse na fase alerta ${ }^{\mathrm{i}}$ & $0=$ sem sinais de estresse $1=$ com sinais de estresse & $\mathrm{j}$ \\
\hline Sinais de estresse na fase resistência ${ }^{\mathrm{i}}$ & $0=$ sem sinais de estresse $1=$ com sinais de estresse & $\mathrm{j}$ \\
\hline Sinais de estresse na fase exaustão ${ }^{i}$ & $0=$ sem sinais de estresse $1=$ com sinais de estresse & $\mathrm{j}$ \\
\hline Atividade física $^{\mathrm{k}}$ & $0=$ ativo $1=$ inativo & 1 \\
\hline \multicolumn{3}{|l|}{ Condições discentes } \\
\hline Turno & $0=$ noturno $1=$ diurno & d \\
\hline Períodos cursados & $0=$ iniciais $1=$ finais & $\mathrm{m}$ \\
\hline \multicolumn{3}{|l|}{ Condições de trabalho } \\
\hline Horas de trabalho por dia & $0=$ até 8 horas $1=$ mais de 8 horas & - \\
\hline 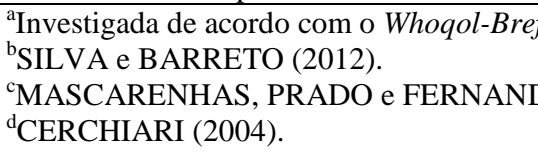 & & \\
\hline \multicolumn{3}{|c|}{$\begin{array}{l}\text { "Investigada de acordo com o "Questionário da Amostra CD 2010" (Instituto Brasileiro de Geografia e Estatística (IBGE, 2010). } \\
\text { f"casado" compreendeu os sujeitos casados e com união estável. "Solteiro/divorciado" envolveu os desquitados ou separados } \\
\text { judicialmente, os divorciados, os viúvos e os solteiros. }\end{array}$} \\
\hline \multicolumn{3}{|c|}{$\begin{array}{l}\text { Investigada de acordo com o Critério de Classificação Econômica Brasil (CCEB). Tais segmentações representam as respectivas } \\
\text { classes de renda familiar: A1: R\$ } 12.926,00 ; \mathrm{A} 2: \mathrm{R} \$ 8.418,00 ; \mathrm{B} 1: \mathrm{R} \$ 4.418,00 ; \mathrm{B} 2: \mathrm{R} \$ 2.565,00 ; \mathrm{C} 1: \mathrm{R} \$ 1.541,00 ; \mathrm{C} 2: \mathrm{R} \$ \\
1.024,00 ; \mathrm{D}: \mathrm{R} \$ 714,00 \text { e E: } \mathrm{R} \$ 477,00 \text {. } \\
{ }^{\mathrm{h}} \mathrm{RECHENCHOSKY} \mathrm{et} \mathrm{al.} \mathrm{(2009).}\end{array}$} \\
\hline \multicolumn{3}{|c|}{$\begin{array}{l}\text { i Investigado de acordo como o Inventário de Sintomas de Stress para Adultos de Lipp -ISSL, validado para uso no Brasil (LIPP, } \\
\text { 2000). }\end{array}$} \\
\hline \multicolumn{3}{|c|}{${ }^{\mathrm{j} S A D I R}$, BIGNOTTO e LIPP (2010). } \\
\hline \multicolumn{3}{|l|}{$\begin{array}{l}{ }^{\mathrm{k}} \text { Investigada de acordo com recomend } \\
{ }^{\mathrm{l}} \text { PUCCI et al (2012). }\end{array}$} \\
\hline
\end{tabular}


A análise estatística se deu pelo software Statistical Package for the Social Sciences v.17.0. Para avaliar associação entre QV e variáveis independentes, realizou-se análise bivariada através do Teste Quiquadrado considerando associação ao nível de $\mathrm{p} \leq 0,20$. Variáveis independentes que se mostraram associadas foram incluídas nas análises de regressão logística múltipla retendo no modelo final aquelas que se associaram com QV ao nível de $\mathrm{p} \leq 0,05$.

Estudo aprovado pelo Comitê de Ética e Pesquisa da SOEBRAS com parecer consubstanciado 274.425 .

\section{RESULTADOS E DISCUSSÃO}

Dos 310 protocolos de pesquisa respondidos, dez $(3,22 \%)$ foram excluídos por apresentarem respostas incoerentes ou incompletas. Destaca-se que apesar de constante busca em bases de dados literárias, ainda são incipientes os estudos que abordaram a relação entre qualidade de vida, estresse e trabalho entre estudantes do ensino superior, o que delimita as discussões desta pesquisa.

A avaliação da percepção do nível de QV geral e por domínios (físico, psicológico, social e ambiental) identificou altos níveis de percepção entre 64,7\% (n=194); 77,0\% $(\mathrm{n}=231) ; 81,7 \% \quad(\mathrm{n}=245) ; 84,0 \% \quad(\mathrm{n}=252)$; $73,0 \% \quad(\mathrm{n}=219)$ respectivamente. Estudos nacionais com estudantes de medicina também registraram altos níveis de percepção do nível de QV. (RAMOS-DIAS, 2010; MEYER et al., 2012). Pesquisa entre estudantes de fisioterapia, tendo utilizado para coleta de dados o questionário SF-36, identificou que a maioria dos domínios apresentou boa percepção da qualidade de vida, exceto o domínio dor. (BACCHI et al., 2013). Estudo prévio identificou, através do Whoqol-Bref, escores significativamente maiores em todos os domínios da QV entre estudantes trabalhadores quando comparados a estudantes que não possuíam atividade remunerada. (CERCHIARI, 2004).

Quanto às variáveis independentes que se mostraram associadas à percepção do baixo nível de QV e foram incluídas nas análises de regressão logística múltipla registou-se que10,7\% (n=32) tem filho; 67,0\% ( $n=201)$ estuda no turno noturno; 50,0\% $(\mathrm{n}=150)$ trabalha; 7,0\% $(n=21)$ apresentou sinais de estresse na fase alerta; 45,0\% $(n=135)$ na fase de resistência e $12,0 \% \quad(n=36)$ na fase de exaustão.

Na Tabela 2 são apresentados os resultados do modelo final. Registrou-se menor chance de baixa percepção no nível de QV geral entre estudantes do turno diurno quando comparados aos estudantes do noturno diurno; e maior chance entre os estudantes com sinais de estresse na fase de resistência quando comparados aos estudantes sem sinais de estresse nesta fase. Maior chance de baixa percepção no nível de QV no domínio físico entre os estudantes com sinais 
de estresse nas fases de resistência e exaustão quando comparados aos estudantes sem sinais de estresse nestas fases. Menor chance de baixa percepção no nível de QV no domínio psicológico entre estudantes que relataram não ter filho quando comparados àqueles que os têm; e maior chance entre estudantes com sinais de estresse na fase de resistência quando comparados aos estudantes sem sinais de estresse nesta fase. Menor chance de baixa percepção no nível de QV no domínio ambiental entre estudantes que não trabalham quando comparados aos que trabalham; e maior chance entre estudantes com sinais de estresse nas fases de alerta e resistência quando comparados aos estudantes sem sinais de estresse nestas fases.

Tabela 2 - Análise de regressão logística múltipla da relação entre qualidade de vida, estresse e trabalho entre estudantes de fisioterapia, Montes Claros - MG, 2013 ( $n=300)$

\begin{tabular}{|c|c|c|c|c|c|c|c|c|c|c|c|c|}
\hline \multirow{2}{*}{ VARIÁVEIS } & \multicolumn{3}{|c|}{ QV Geral } & \multicolumn{3}{|c|}{ Domínio Físico } & \multicolumn{3}{|c|}{ Domínio Psicológico } & \multicolumn{3}{|c|}{ Domínio Ambiental } \\
\hline & OR & $\mathrm{IC}_{\mathbf{9 5} \%}$ & $\mathbf{p}$ & OR & $\mathrm{IC}_{\mathbf{9 5} \%}$ & $\mathbf{p}$ & $\mathbf{O R}$ & $\mathrm{IC}_{95 \%}$ & $\mathbf{p}$ & $\mathbf{O R}$ & $\mathrm{IC}_{95 \%}$ & $\mathbf{p}$ \\
\hline Filhos & & & & & & & & & & & & \\
\hline Sim & -- & -- & -- & -- & -- & -- & 1,00 & & & -- & -- & -- \\
\hline Não & -- & -- & -- & -- & -- & -- & 0,33 & $0,14-0,77$ & 0,011 & -- & -- & -- \\
\hline \multicolumn{13}{|l|}{ Turno } \\
\hline Noturno & 1,00 & & & -- & -- & -- & -- & -- & -- & -- & -- & -- \\
\hline Diurno & 0,54 & $0,32-0,92$ & 0,025 & -- & -- & -- & -- & -- & -- & -- & -- & -- \\
\hline Sim & -- & -- & -- & -- & -- & -- & -- & -- & -- & 1,00 & & \\
\hline Não & -- & -- & -- & -- & -- & -- & -- & -- & -- & 0,51 & $0,30-0,87$ & 0,014 \\
\hline \multicolumn{13}{|c|}{ Estresse (alerta) } \\
\hline Não & -- & -- & -- & -- & -- & -- & -- & -- & -- & 1,00 & & \\
\hline Sim & -- & -- & -- & -- & -- & -- & -- & -- & -- & 2,96 & $1,13-7,75$ & 0,028 \\
\hline \multicolumn{13}{|c|}{ Estresse (resistência) } \\
\hline Não & 1,00 & & & 1,00 & & & 1,00 & & & 1,00 & & \\
\hline Sim & 2,04 & $1,26-3,32$ & 0,004 & 2,87 & $1,54-5,35$ & 0,001 & 4,40 & $2,28-8,51$ & 0,000 & 1,97 & $1,13-3,44$ & 0,017 \\
\hline Não & -- & -- & -- & 1,00 & & & -- & -- & -- & -- & -- & -- \\
\hline Sim & -- & -- & -- & 2,77 & $1,27-6,05$ & 0,010 & -- & -- & -- & -- & -- & -- \\
\hline
\end{tabular}

A identificação de menor chance de baixa percepção no nível de QV geral entre estudantes do turno diurno diverge com estudos nacionais envolvendo estudantes do ensino superior onde se registraram que os escores de percepção no nível de QV geral dos acadêmicos de tempo integral são significativamente menores em relação aos do período noturno, (OLIVEIRA, 1999), sendo tal percepção significativamente menor entre estudantes de turno integral, seguidos pelos do turno matutino e por fim daqueles do turno noturno. (CERCHIARI, 2004). Plausibilidade razoável para tal registro é o fato de que a maioria dos estudantes do turno noturno trabalha e a dupla jornada de serviço pode vir a impactar na qualidade de vida dos estudantes e consequentemente afetar o estado de saúde. (TOMBOLATO, 2005).

A baixa percepção do nível à QV geral bem como dos domínios físico, psicológico e ambiental se associaram à fase de resistência do estresse, caracterizada pelo enfrentamento ou adaptação ao agente estressor. (SADIR et al., 2010). Resultado divergente de pesquisa realizada com estudantes de medicina de 
Santa Catarina, que identificou associações muito fracas entre os domínios da $\mathrm{QV}$ e as dimensões da Job Stress Scale (demanda psicológica, controle e apoio social), não havendo diferença significativa entre elas. (MEYER et al., 2012).

O domínio físico da QV compreende aspectos como energia e fadiga, dor e desconforto, sono e repouso. (TABELEÃO, TOMASI, NEVES, 2011). Acredita-se que sua relação à fase de resistência de estresse possa ser justificada pela grande quantidade de energia despendida na elaboração de estratégias de enfrentamento dos agentes estressores, de forma a evitar maior enfraquecimento físico e posterior desenvolvimento de doenças. Nesta fase, predominam indicadores de estresse de natureza física e a pessoa pode desenvolver quadros de cefaleia, tensão muscular e desgaste físico constante dentre outros. (SANTOS e CARDOSO, 2010).

Baixa percepção do nível de QV no domínio físico também se relacionou à fase de exaustão do estresse na qual, a pessoa deixa de enfrentar os agentes estressores e passa a apresentar sintomas com maior dimensão como intensa exaustão física e psicológica em forma de transtorno depressivo. (SILVA E MARTINEZ, 2005).

O domínio psicológico permeia sentimentos positivos, pensamento, aprendizagem, memória e concentração, autoestima, imagem corporal e aparência e sentimentos negativos. (TABELEÃO, TOMASI, NEVES, 2011). Sua relação à fase de resistência possivelmente fundamenta-se no fato de que, a tendência da pessoa se estressar, associa-se a pensamentos errôneos ou distorcidos, bem como às avaliações e interpretações erradas sobre os eventos da vida; ao contexto da formação acadêmica delineado por exigência de alto desempenho, concentração e esforços; ao temor da não aprovação por docentes e pares diante tomada de decisão inadequada, com consequente comprometimento da autoestima e sentimentos de falta de controle, fracasso, desesperança, ansiedade, raiva e desespero. (LIPP, 2001; NOGUEIRA-MARTINS, 2002; FURTADO, FALCONE e CLARK, 2003; ASSIS et al., 2013). Tais condições podem levar o estudante à busca pelo reequilíbrio com consequente desgaste sem causa aparente e ainda ao comprometimento da memória. (ASSIS et al., 2013).

Não se encontrou na literatura estudos que corroboram com o resultado identificado na presente pesquisa acerca do registro de menor chance de baixa percepção no nível de QV no domínio psicológico entre estudantes que não têm filhos quando comparado aos que têm. Entretanto, tal resultado diverge de estudo conduzido entre professores no qual se verificou que os profissionais sem filhos possuíam maiores níveis nas dimensões do Maslach Burnout Inventory (exaustão 
emocional, despersonalização e realização profissional). (CARLOTTO, 2011).

O domínio ambiental envolve segurança física e proteção, ambiente no lar, recursos financeiros, disponibilidade e qualidade de cuidados de saúde e sociais, oportunidades de adquirir novas informações e habilidades, oportunidades e participação em atividades de recreação e lazer, ambiente físico e transporte. (TABELEÃO, TOMASI, NEVES, 2011). Acredita-se que sua relação à fase de resistência possa ser justificada pela exposição aos agentes estressores externos com consequentes tentativas de adaptação às mudanças do ambiente no qual se encontra inserido. (LIPP, 2001). Neste contexto, no ambiente acadêmico o estudante pode estar exposto a pressões desencadeadoras de estresse, uma vez que aí vivencia momentos de mudança, desenvolvimento, frustração, crescimento, temores, angústias, bem como a constante necessidade de resolução de problemas. (MONTEIRO, FREITAS e RIBEIRO, 2007). Mudanças no ambiente externo à instituição de ensino também podem levar o estudante a uma constante busca pela readaptação uma vez que este pode mostrar-se insatisfeito com as condições do local onde reside, com a acessibilidade aos serviços de saúde e transporte, com dificuldades financeiras e com as situações de insegurança e violência vivenciadas pelo jovem na sociedade. (COSTA et al., 2008).
Baixa percepção do nível de QV no domínio ambiental também se relacionou à primeira fase do estresse, compreendida como fase de alerta, na qual a pessoa necessita despender esforço e energia para executar exigências imposta e enfrentar situações desafiadoras. (SILVA E MARTINEZ, 2005). Contudo, esta fase é tida como benéfica uma vez que prepara a pessoa para enfrentar adversidades e restabelecer o equilíbrio. Todavia se o agente estressor persistir, a pessoa passa à segunda fase -resistência(PACANARO e SANTOS,2007).

$\mathrm{O}$ registro de menor chance de baixa percepção no nível de QV no domínio ambiental entre estudantes que não trabalham diverge de estudo prévio que identificou relação entre "não ter trabalho remunerado" e impacto negativo na percepção do nível de QV de estudantes universitários, uma vez que esta se associaria a questões socioeconômicas e recursos financeiros, à qualidade de cuidados de saúde e sociais, e à participação em atividades de recreação e lazer. (CERCHIARI, 2004; TABELEÃO, TOMASI, NEVES, 2011).

\section{CONCLUSÃO}

Registrou-se menor chance de baixa percepção no nível de QV geral entre estudantes do turno diurno e maior chance entre os estudantes com sinais de estresse na fase de resistência. Maior chance de baixa percepção no nível de QV no domínio físico 
entre os estudantes com sinais de estresse nas fases de resistência e exaustão. Menor chance de baixa percepção no nível de QV no domínio psicológico entre estudantes que relataram não ter filho e maior chance entre estudantes com sinais de estresse na fase de resistência. Menor chance de baixa percepção no nível de QV no domínio ambiental entre estudantes que não trabalham e maior chance entre estudantes com sinais de estresse nas fases de alerta e resistência.

\section{REFERÊNCIAS}

ASSIS, C. L. et al. Sintomas de estresse em concluintes do curso de psicologia de uma faculdade privada do norte do País.

Mudanças - Psicologia da Saúde, São

Paulo, v. 21, n. 1, p. 23-28, 2013.

BACCHI, C. A. et al. Avaliação da qualidade de vida, da dor nas costas, da funcionalidade e de alterações da coluna vertebral de estudantes de fisioterapia. Motriz: revista de educação física, Rio Claro, v. 19, n. 2, p. 243-251, 2013.

BOTTI, N. C. L. LIMA, A. F. D. SIMÕES, W. M. B. Uso de substâncias psicoativas entre acadêmicos de enfermagem da Universidade Católica de Minas Gerais. Rev. Eletrônica Saúde Mental Álcool Drogas, Ribeirão Preto, v. 6, n. 1, p. 6 (1): 1-13, 2010.

\section{BRASIL. Ministério da Saúde. Vigitel Brasil}

2009: vigilância de fatores de risco e proteção para doenças crônicas por inquérito telefônico. Ministério da Saúde, Secretaria de Vigilância em Saúde, Secretaria de Gestão Estratégica e Participativa. Brasília:

Ministério da Saúde; 2010.

BUBLITZ, S. et al. Estresse em estudantes de enfermagem: uma revisão integrativa. Revista de Enfermagem UFSM, Santa Maria, v. 2, n. 3, p. 530-538, 2012.

CALAIS, S. L. et al. Estresse entre calouros e veteranos de jornalismo. Estudos de Psicologia, Campinas, v. 24, n. 1, p. 69-77, 2007.

CARLOTTO, M. S. Síndrome de Burnout em professores: prevalência e fatores associados. Psicologia Teoria e Pesquisa, Brasília, v. 27, n. 4, p. 403-410, 2011.

CERCHIARI, E. A. N. Saúde mental e qualidade de vida em estudantes universitários. Originalmente apresentada como tese de doutorado. Faculdade de Ciências Médicas - Universidade Estadual de Campinas, Campinas, 2004.

COSTA, C. C et al. Qualidade de Vida e Bem-Estar Espiritual los Universitários de Psicologia. Psicologia em Estudo, Maringá, v.13, n.2, p. 249-255, 2008.

\section{FERNANDES, E. C. Qualidade de vida no}

trabalho: como medir para melhorar.

Salvador: Casa da Qualidade; 1996. 
FLECK, M. P. A. et al. Aplicação da versão em português do instrumento abreviado de avaliação da qualidade de vida "WHOQOLbref”. Revista de Saúde Pública, São Paulo, v. 34, n. 2. p.178-183, 2000.

FURTADO, E. S. FALCONE, E. M. O.

CLARK C. Avaliação do estresse e das habilidades sociais na experiência acadêmica de estudantes de medicina de uma Universidade do Rio de Janeiro. Interação em Psicologia, Curitiba, v. 7, n. 2, p. 43-51, 2003.

\section{LIPP, M. Manual do Inventário de}

Sintomas de Stress para adultos de Lipp

(ISSL). São Paulo: Casa do Psicólogo; 2000.

LIPP, M. E. N. Estresse emocional: a contribuição de estressores internos e externos. Revista de Psiquiatria Clínica, v. 28, n. 6, p. 347-349, 2001.

MACIEL, E. S. Qualidade de vida: análise da influência do consumo de alimentos e estilo de vida. Originalmente apresentada como dissertação de mestrado, Escola Superior de Agricultura Luiz de Queiroz, Universidade de São Paulo, 2006.

MASCARENHAS, C. H. M. PRADO, F. O. FERNANDES, M. H. Fatores associados à qualidade de vida de Agentes Comunitários de Saúde. Ciência e Saúde Coletiva, Rio de Janeiro, v.18, n.5, p. 1375-1386, 2013.

MEYER, C. et al. Qualidade de vida e estresse ocupacional em estudantes de medicina. Revista Brasileira de Educação Médica, Rio de Janeiro, v. 36, n. 4, p. 489498, 2012.

MINAYO, M. C. S. HARTZ, Z. M. A. BUSS, P. M. Qualidade de vida e saúde: um debate necessário. Ciência e Saúde Coletiva, Rio de Janeiro, v. 5, n. 1, p. 7-18, 2000.

NOGUEIRA-MARTINS, M. C. F. (2002).

Humanização das relações assistenciais: a formação do profissional de saúde. São Paulo: Casa do Psicólogo.

OLIVEIRA, J. A. C. Qualidade de vida em estudantes universitários de Educação Física. Originalmente apresentada como dissertação de mestrado, Faculdade de Ciências Médicas da Unicamp, Campinas, 1999.

PACANARO, S. V. SANTOS, A. P. A. Avaliação do estresse no contexto educacional: análise de produção de artigos científicos. Avaliação Psicológica, Itatiba, v. 6, n. 2, p. 253-260, 2007. 
PUCCI, G. C. M. F. et al. Associação entre atividade física e qualidade de vida em adultos. Revista de Saúde Pública, São Paulo, v. 46, n. 1, p. 166-179, 2012.

RAMOS-DIAS, J. C. et al. Qualidade de vida em cem alunos do curso de Medicina de Sorocaba - PUC/SP. Revista Brasileira de Educação Médica, Rio de Janeiro, v. 34, n. 1, p. 116-123, 2010.

RECHENCHOSKY, L. et al. Estado nutricional e perfil lipídico de crianças. Revista da Educação Física/UEM, Maringá, v. 20, p. 431-440, 2009.

SADIR, M. A. BIGNOTTO, M. M. LIPP M. E. N. Stress e qualidade de vida: influência de algumas variáveis pessoais. Paidéia, Ribeirão Preto, v. 20, n.45, p. 73-81, 2010.

SANTOS, A. F. O. CARDOSO, C. L.

Profissionais de saúde mental: estresse, enfrentamento e qualidade de vida.

Psicologia: Teoria e Pesquisa, Brasília, v. 26, n. 3, p. 543-548, 2010.

SILVA, E. A. T. MARTINEZ, A. Diferença em nível de stress em duas amostras: capital e interior do estado de São Paulo. Estudos de Psicologia, Campinas, v. 22, n. 1, p. 53-61, 2005.
SILVA, L. S. BARRETO, S. M. Adverse psychosocial working conditions and poor quality of life among financial service employees in Brazil. Journal of Occupational Health, Bethesda, v. 54, p. 88-95, 2012.

SILVA, R. V. SANTOS, S. S. C. 2000. O cotidiano de alunas de enfermagem após início do estágio [Resumo]. Associação Brasileira de Enfermagem (Org.), Resumos de comunicações científicas, $53^{\circ}$ Congresso Brasileiro de Enfermagem. CD Rom. Curitiba. PR.

TABELEÃO, V. P. TOMASI, E. NEVES, S.

F. Qualidade de vida e esgotamento profissional entre docentes da rede pública de Ensino Médio e Fundamental no Sul do Brasil. Cadernos de Saúde Pública, Rio de Janeiro, v. 27, n. 12, p. 2401-2408, 2011.

\section{TOMBOLATO, M. C. R. Qualidade de} vida e sintomas psicopatológicos do estudante trabalhador. Originalmente apresentada como dissertação de mestrado, Pontifícia Universidade Católica de Minas Gerais, Belo Horizonte, 2005.

WORLD HEALTH ORGANIZATION. Global strategy on diet, physical activity and health. Geneva: WHO, 2004. 